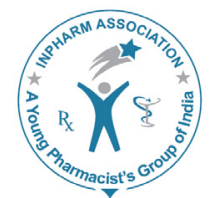

\title{
Evaluation of novel 1-(4-(substituted)piperazin-1-yl)- 2-(phenylamino)ethanone derivatives as Falcipain-2 inhibitors
}

\author{
Sourabh Mundra and Radhakrishnan Mahesh \\ Department of Pharmacy, FD-III, Birla Institute of Technology \& Science, Pilani, Rajasthan-333031, India.
}

\begin{abstract}
Background: Malaria, an infectious disease transmitted by mosquitoes, has affected the world since the beginning of recorded human history and it remains an ensconced global health challenge even today. Among the various proteases, expressed in the life cycle of parasite, cysteine protease falcipain-2 plays a pivotal role in parasite food assimilation and inhibition of this protease cause deleterious effects on the growth of parasite. Methods: Employing a ligand-based approach, 1-(4-(substituted)piperazin-1-yl)-2-(phenylamino) ethanone derivatives were designed and synthesized from the starting material piperazine in a sequence of reactions. Structural assignments are based on spectral data ( ${ }^{1} \mathrm{H}$ NMR, mass) and elemental analyses. The purity of the final compounds was confirmed by HPLC. The compounds were tested for their in vitro falcipain-2 inhibitor activity on recombinant falcipain-2 enzyme. Furthermore, molecular docking studies were performed using Glide 5.9 software to incur a precise picture of the active ligand at the atomic level which will be helpful in the discovery of new antimalarial drugs. Results: Among the screening results of seventeen novel entities, three compounds ( $6 \mathrm{~h}, 6 \mathrm{n}$ and $60)$ have showed good inhibitory activity and eleven compounds were showed weak to moderate inhibitor activity. Docking studies for these active analogues revealed that the amino acids Trp 206, Ile 85, Leu 84, Val 152 most commonly involved in hydrophobic interactions and Asn 173, Cys 42, Gln 36, amino acids involved in hydrogen bonding. Conclusion: The preliminary structure-activity relationships indicated that compound $6 \mathrm{~h}$, is the most potent compound from this series, and it can be used as a potential lead compound in the designing of new candidates to optimize the inhibitory potencies of this class of compounds, and potentially with potent antimalarial activity.
\end{abstract}

Key words: Antimalarial, Falcipain-2, Ligand-based drug design, Plasmodium falciparum.

\section{INTRODUCTION}

\begin{tabular}{|c|c|}
\hline \multicolumn{2}{|c|}{ Access this article online } \\
\hline Journal Sponsor & \multirow[b]{2}{*}{$\begin{array}{l}\text { Website: } \\
\text { www.jyoungpharm.org }\end{array}$} \\
\hline & \\
\hline www.phco & DOI: 10.5530/jyp.2015.2.7 \\
\hline
\end{tabular}

Malaria an infectious disease caused by Plasmodium parasites, among which Plasmodium falciparum is the most dangerous one, with the highest rates of complications such as cerebral malaria or severe anemia and mortality. There has never been a time when new antimalarial drugs were needed more, with an estimated 3.4 billion people are at risk of malaria, of whom 1.2 billion are at high risk. In high-risk areas, more than one malaria case occurs per 1000

\footnotetext{
*Address for correspondence:

Sourabh Mundra, Senior Research Scholar, Pharmacy Department, Birla Institute of science \& Technology (BITS) Pilani-333031,

Rajasthan, India. Email: sourabh_mundra@yahoo.co.in
} 
population. ${ }^{1}$ In 2012 , malaria killed 1300 children every day or one child almost every minute underage five. ${ }^{1}$ Control of malaria has been severely conciliated by the development of malaria parasites resistant to nearly all available antimalarial drugs used for prophylaxis and treatment, particularly in Plasmodium falciparum. Parasite resistance towards Artemisinin the key compounds in Artemisinin combination therapies (ACTs) is also emerging ${ }^{2,3}$ in at least four countries of the South-East Asia region. The rapid re-emergence of resistance to all available drugs made malaria a more acute problem, hence represents a severe limitation of effective drugs in many high-risk areas. Thus, drug resistance to all existing antimalarial drugs is a cause for immediate concern. Therefore, development of novel and effective chemical class of antimalarial drugs especially compounds that act against novel biochemical targets is the better approach to deceive the problem faced by clinically used drugs. To develop such compounds, it is very important to elucidate the structural and biochemical features of new drug targets.

In the class of targets for antimalarial chemotherapy, the cysteine proteases of Plasmodium falciparum is an attractive and promising target. ${ }^{4,5}$ Proteases are druggable targets, and various protease inhibitors are now licensed as well as in clinical phase to treat different diseases for example osteoporosis, diabetes, cancer, hypertension, and infectious diseases. ${ }^{6-9}$ Among the four P. falciparum cysteine proteases, falcipain- 2 is the most intensely studied enzyme and it appears to be the essential food vacuolar hemoglobinases. ${ }^{10-12}$ Studies affirmed that when falcipain-2 gene is disrupted, undegraded hemoglobin accumulates in the food vacuole, confirming that this enzyme participates in digestion of hemoglobin in the acidic food vacuole. ${ }^{13,14}$ Various research groups have investigated the cysteine protease inhibitors, which are mainly originated from peptides derivatives ${ }^{15,16}$ and having nanomolar $\mathrm{IC}_{50}$ values, due to the formation of covalent bond with thiol of active site Cys42, which behaves as a Michael acceptor. ${ }^{17}$ The poor selectivity for parasitic cysteine proteases over the human cysteine proteases remains a noteworthy concern.

This discussion emphasizes the demand for peptidomimetic or non-peptidic analogues that would bind non-covalently to the target protein in order to reduce the toxicity, while retaining the high degree in-vivo activity and selectivity. Over the past many years, our group identified potent antimalarial targeting orotidine-5'-monophosphate decarboxylase $(\text { ODCase })^{18}$ paving the way toward non-covalent antimalarial therapeutic agents. Herein, we report design, synthesis and in-vitro evaluation of a novel series of 1-(4-(substituted) piperazin-1-yl)-2-(phenylamino) ethanone derivatives 6(a- q) as potential non-covalent inhibitors of cysteine protease falcipain-2. Further, we utilized docking tools for the most active compounds to investigate their interactions and binding affinities in the falcipain-2 active site.

\section{MATERIALS AND METHODS}

Buchi 530 melting point apparatus (open capillary tubes), was used to compute melting points and were uncorrected. Reaction progress was monitored by thin layer chromatography (Silica gel-60 $\mathrm{F}_{254}$ ). Compounds were detected by their absorption under UV light. Bruker DPX spectrometer operating at $400 \mathrm{MHz}$ in $\mathrm{CDCl}_{3}$ or DMSO- $d_{6}$ solvent, with tetramethylsilane (TMS) as an internal standard, was used to record ${ }^{1} \mathrm{H}$ NMR spectra. Chemical shifts were reported in $\delta \mathrm{ppm}$; the $J$ values are expressed in Hertz $(\mathrm{Hz})$. Purity of the synthesized final compounds was determined by Waters ${ }^{\mathrm{TM}} \mathrm{LC}-\mathrm{MS}$ system using a XBridge semipreparative $C_{18}$ column (19.2 $\mathrm{mm} \mathrm{x}$ $150 \mathrm{~mm}, 5 \mu \mathrm{m}$ ). Mass spectra (ESI) were recorded on a Waters $^{\mathrm{TM}}$ LC/MS system equipped with a Waters ${ }^{\mathrm{TM}} 3100$ mass detector. Elemental analyses were performed on a PE-2400 elemental analyzer; the $\mathrm{C}, \mathrm{H}$ and $\mathrm{N}$ analysis were repeated duplicate. All solvents and reagents were obtained commercially from Aldrich, Fluka, Spectrochem and S.D. fine.

\section{Synthesis of N-Boc-piperazine (2)}

In a $500 \mathrm{ml}$ round bottom flask, compound $1(10 \mathrm{~g}, 116.1$ $\mathrm{mmol})$ was dissolved in methanol $(100 \mathrm{~mL})$. To this, water $(2 \mathrm{~mL})$ and a solution of trifluoroacetic acid $(8.9 \mathrm{~mL}, 116.1$ $\mathrm{mmol})$ in DCM $(10 \mathrm{~mL})$ were added at $0-5^{\circ} \mathrm{C}$; the reaction mixture was stirred for $30 \mathrm{~min}$ at room temperature. To the above solution, di-tert-butyldicarbonate $(26.6 \mathrm{~mL}, 116.1$ $\mathrm{mmol})$ was added followed by iodine $(10 \mathrm{~mol} \%)$. The reaction mixture was again stirred at room temperature for $3 \mathrm{~h}$; On completion of the reaction, solvent was removed under vacuum. To the obtained residue, $5 \%$ sodium thiosulphate solution $(15 \mathrm{~mL})$ was added and extracted with ethyl acetate $(2 \times 50 \mathrm{~mL})$. The organic layer was dried over anhydrous sodium sulfate and concentrated. The crude compound 2 was purified by flash chromatography and pure product obtained as a colourless liquid. Yield: $60 \%$, ${ }^{1} \mathrm{H}$ NMR (400 MHz, DMSO- $\left.d_{6}\right) \delta: 3.22(\mathrm{~m}, 4 \mathrm{H}), 2.61(\mathrm{~m}$, $4 \mathrm{H}), 1.38$ (s, 9H).

\section{Synthesis of tert-butyl 4-(2-chloroacetyl) piperazine-1- carboxylate (3)}

To a solution of 2 ( $4 \mathrm{~g}, 21.4 \mathrm{mmol})$, di-isopropylethylamine $(5.6 \mathrm{~mL}, 32.2 \mathrm{mmol})$ in anhydrous DCM $(200 \mathrm{~mL})$ were taken in a round bottom flask $(500 \mathrm{~mL})$ at $0^{\circ} \mathrm{C}$. To this 
reaction mixture, chloroacetyl chloride $(1.7 \mathrm{~mL}, 21.4 \mathrm{mmol})$ was added. The reaction mixture was stirred at room temperature for $30 \mathrm{~min}$; quenched with saturated sodium hydrogen carbonate $(10 \mathrm{~mL})$ and washed with water $(2 \mathrm{x}$ $50 \mathrm{~mL}$ ). The organic layer was separated and dried over sodium sulfate followed by evaporation of the solvent to get a crude compound 3; which was purified by column chromatography and obtained as a white solid. Yield: $70 \%$, ${ }^{1} \mathrm{H}$ NMR (400 MHz, DMSO-d $)$ ) 8.39 (s, 2H), 3.44 (m, 4H), 3.32 (s, 4H), 1.40 (s, 9H).

\section{Synthesis of tert-butyl 4-(2-(phenylamino)acetyl) piperazine-1-carboxylate (4)}

To a solution of compound $3(2.5 \mathrm{~g}, 9.5 \mathrm{mmol})$, in anhydrous acetonitrile $(25 \mathrm{~mL}), \mathrm{K}_{2} \mathrm{CO}_{3}(3.9 \mathrm{~g}, 28.6 \mathrm{mmol})$, and aniline $(0.95 \mathrm{ml}, 10.4 \mathrm{mmol})$ were added. The reaction mixture was refluxed for $3 \mathrm{~h}$ at $100^{\circ} \mathrm{C}$; completion of the reaction was monitored by TLC. Solvent was removed under vacuum, and organic compound was extracted with ethyl acetate $(2 \times 25 \mathrm{~mL})$. The combined organic extracts were dried over sodium sulphate and evaporated to get crude compound 4, which was purified by column chromatography and obtained as a colourless liquid. Yield: 65\%, ${ }^{1} \mathrm{H}$ NMR $\left(400 \mathrm{MHz}, \mathrm{DMSO}-d_{\mathrm{c}}\right) \delta: 7.07(\mathrm{~m}, 2 \mathrm{H}), 6.57$ $(\mathrm{m}, 2 \mathrm{H}), 6.46(\mathrm{~d}, J=6 \mathrm{~Hz}, 1 \mathrm{H}), 4.12(\mathrm{~s}, 2 \mathrm{H}), 3.65(\mathrm{~m}, 4 \mathrm{H})$, $3.12(\mathrm{~m}, 4 \mathrm{H}), 1.42(\mathrm{~s}, 9 \mathrm{H})$.

\section{Synthesis of 2-(phenylamino)-1-(piperazin-1-yl) ethanone (5)}

To a solution of 4 ( $1 \mathrm{~g}, 3.1 \mathrm{mmol})$ in anhydrous DCM (20 $\mathrm{mL}$ ) in a round bottom flask at $0^{\circ} \mathrm{C}$, trifluroacetic acid $(1.94 \mathrm{~mL}, 25.1 \mathrm{mmol})$ was added. The reaction mixture was stirred at room temperature for $7 \mathrm{~h}$. Upon completion of the reaction, solvent was removed under vacuum. To the resultant crude, saturated solution of sodium bicarbonate $(10 \mathrm{~mL})$ was added and extracted with ethyl acetate $(2 \times 50$ $\mathrm{mL})$. Ethyl acetate portion was evaporated to obtain the free amine 5, as off white solid. Yield: 70\%, ${ }^{1} \mathrm{H}$ NMR (400 $\left.\mathrm{MHz}, \mathrm{DMSO}-d_{6}\right) \delta: 8.53$ (br. s, IH), 7.07 (m, 2H), 6.57 (m, 2H), $6.46(\mathrm{~d}, J=6 \mathrm{~Hz}, 1 \mathrm{H}), 5.55(\mathrm{~d}, J=4.0 \mathrm{~Hz}, 1 \mathrm{H}), 3.93$ (s, 2H), $3.65(\mathrm{~m}, 4 \mathrm{H}), 3.12(\mathrm{~m}, 4 \mathrm{H})$.

General procedure for the synthesis of 1-(4-(substituted) piperazin-1-yl)-2-(phenylamino)ethanone derivatives 6(a-q)

To an appropriate quantity of carboxylic acid $(1 \mathrm{~g})$, TEA (2.4 equiv), $\mathrm{EDC} \cdot \mathrm{HCl}$ (1.5 equiv), and $\mathrm{HOBt}(0.8$ equiv) in anhydrous DCM were stirred for $5 \mathrm{~min}$ at $0^{\circ} \mathrm{C}$, followed by the addition of compound 5 (1.1 equiv). The mixture was stirred for $6 \mathrm{~h}$ at room temperature, solvents removed under reduced pressure. The residue was dissolved in ethyl acetate, and the organic phase was washed with saturated sodium bicarbonate (twice) and brine solution (once). The organic layer was dried over anhydrous sodium sulfate and evaporated under reduced pressure, and the obtained crude product was purified by column chromatography.

\section{1-(4-Benzoylpiperazin-1-yl)-2-(phenylamino)ethanone} (6a)

${ }^{1} \mathrm{H}$ NMR $\left(\mathrm{CDCl}_{3}\right)$ 8: $7.39(\mathrm{~m}, 2 \mathrm{H}), 7.34(\mathrm{~m}, 3 \mathrm{H}), 7.13(\mathrm{t}$, $J=7.8 \mathrm{~Hz}, 2 \mathrm{H}), 6.67(\mathrm{t}, J=5.2 \mathrm{~Hz}, 1 \mathrm{H}), 6.56(\mathrm{~d}, J=7.6$ $\mathrm{Hz}, 2 \mathrm{H}), 3.85(\mathrm{~s}, 2 \mathrm{H}), 3.64(\mathrm{~m}, 4 \mathrm{H}), 3.41(\mathrm{~m}, 4 \mathrm{H})$. Anal. calcd for $\mathrm{C}_{19} \mathrm{H}_{21} \mathrm{~N}_{3} \mathrm{O}_{2} \mathrm{C}, 70.57 ; \mathrm{H}, 6.55 ; \mathrm{N}, 12.99$; found C, 70.42; H, 6.49; N, 12.95. MS (ESI): m/z $324.1(\mathrm{M}+1)^{+}$.

1-(4-(2-Fluorobenzoyl)piperazin-1-yl)-2-(phenylamino) ethanone (6b)

${ }^{1} \mathrm{H}$ NMR $\left(\mathrm{CDCl}_{3}\right)$ ): 7.47 (m, 2H), $7.24(\mathrm{~m}, 3 \mathrm{H}), 7.15$ (t, $J$ $=8.6 \mathrm{~Hz}, 1 \mathrm{H}), 6.77(\mathrm{~m}, 1 \mathrm{H}), 6.62(\mathrm{~m}, 2 \mathrm{H}), 3.98(\mathrm{~s}, 2 \mathrm{H})$, $3.90(\mathrm{~m}, 4 \mathrm{H}), 3.55(\mathrm{~m}, 4 \mathrm{H})$. Anal. calcd for $\mathrm{C}_{19} \mathrm{H}_{20} \mathrm{FN}_{3} \mathrm{O}_{2}$ C, 66.85; H, 5.91; N, 12.31; found C, 66.72; H, 5.85; N, 12.35. MS (ESI): $\mathrm{m} / \mathrm{z} 342.2(\mathrm{M}+1)^{+}$.

\section{1-(4-(4-Fluorobenzoyl)piperazin-1-yl)-2-(phenylamino) ethanone (6c)}

${ }^{1} \mathrm{H}$ NMR $\left(\mathrm{CDCl}_{3}\right) \delta: 7.44(\mathrm{~m}, 2 \mathrm{H}), 7.22(\mathrm{t}, J=7.8 \mathrm{~Hz}, 2 \mathrm{H})$, $7.14(\mathrm{~m}, 2 \mathrm{H}), 6.76(\mathrm{t}, J=7.2 \mathrm{~Hz}, 1 \mathrm{H}), 6.65(\mathrm{~d}, J=7.8 \mathrm{~Hz}$, $2 \mathrm{H}), 3.94(\mathrm{~s}, 2 \mathrm{H}), 3.73(\mathrm{~m}, 5 \mathrm{H}), 3.55(\mathrm{~m}, 3 \mathrm{H})$. Anal. calcd for $\mathrm{C}_{19} \mathrm{H}_{20} \mathrm{FN}_{3} \mathrm{O}_{2} \mathrm{C}, 66.85 ; \mathrm{H}, 5.91 ; \mathrm{N}, 12.31$; found $\mathrm{C}$, 66.77; H, 5.94; N, 12.34. MS (ESI): m/z $342.2(\mathrm{M}+1)^{+}$.

1-(4-(4-Chlorobenzoyl)piperazin-1-yl)-2-(phenylamino) ethanone (6d)

${ }^{1} \mathrm{H}$ NMR $\left(\mathrm{CDCl}_{3}\right) \delta: 7.43(\mathrm{~m}, 4 \mathrm{H}), 7.23(\mathrm{t}, J=7.4 \mathrm{~Hz}$, $2 \mathrm{H}), 6.78(\mathrm{t}, J=7.6 \mathrm{~Hz}, 1 \mathrm{H}), 6.67(\mathrm{~d}, J=4.0 \mathrm{~Hz}, 2 \mathrm{H})$, $3.95(\mathrm{~s}, 2 \mathrm{H}), 3.73(\mathrm{~m}, 1 \mathrm{H}), 3.50(\mathrm{~m}, 7 \mathrm{H})$. Anal. calcd for $\mathrm{C}_{19} \mathrm{H}_{20} \mathrm{ClN}_{3} \mathrm{O}_{2} \mathrm{C}, 63.77 ; \mathrm{H}, 5.63 ; \mathrm{N}, 11.74$; found $\mathrm{C}, 63.78$; $\mathrm{H}$, 5.64; N, 11.71. MS (ESI): $\mathrm{m} / \mathrm{z} 357.1(\mathrm{M})^{+}$and 358.2 $(\mathrm{M}+1)^{+}$.

\section{2-(Phenylamino)-1-(4-(4-(trifluoromethyl)benzoyl)} piperazin-1-yl)ethanone (6e)

${ }^{1} \mathrm{H} \mathrm{NMR}\left(\mathrm{CDCl}_{3}\right)$ ): $7.75(\mathrm{~d}, J=8.0 \mathrm{~Hz}, 2 \mathrm{H}), 7.57(\mathrm{~m}, 2 \mathrm{H})$, $7.23(\mathrm{t}, J=8.0 \mathrm{~Hz}, 2 \mathrm{H}), 6.78(\mathrm{t}, J=7.2 \mathrm{~Hz}, 1 \mathrm{H}), 6.68(\mathrm{~m}$, $2 \mathrm{H}), 3.96(\mathrm{~s}, 2 \mathrm{H}), 3.71(\mathrm{~m}, 4 \mathrm{H}), 3.51(\mathrm{~m}, 4 \mathrm{H})$. Anal. calcd for $\mathrm{C}_{20} \mathrm{H}_{20} \mathrm{~F}_{3} \mathrm{~N}_{3} \mathrm{O}_{2} \mathrm{C}, 61.38 ; \mathrm{H}, 5.15 ; \mathrm{N}, 10.74$; found $\mathrm{C}$, 61.37; H, 5.14; N, 10.71. MS (ESI): m/z $392.1(\mathrm{M}+1)^{+}$.

\section{1-(4-(2-Methylbenzoyl)piperazin-1-yl)-2-(phenylamino) ethanone (6f)}

${ }^{1} \mathrm{H} \mathrm{NMR}\left(\mathrm{CDCl}_{3}\right)$ 8: $7.25(\mathrm{~m}, 1 \mathrm{H}), 7.17(\mathrm{~m}, 3 \mathrm{H}), 7.13(\mathrm{t}, J$ $=7.8 \mathrm{~Hz}, 2 \mathrm{H}), 6.69(\mathrm{~m}, 1 \mathrm{H}), 6.57(\mathrm{~m}, 2 \mathrm{H}), 3.89(\mathrm{~s}, 2 \mathrm{H})$, $3.78(\mathrm{~m}, 2 \mathrm{H}), 3.32(\mathrm{~m}, 6 \mathrm{H}), 2.17(\mathrm{~s}, 3 \mathrm{H})$. Anal. calcd for $\mathrm{C}_{20} \mathrm{H}_{23} \mathrm{~N}_{3} \mathrm{O}_{2} \mathrm{C}, 71.19$; $\mathrm{H}, 6.87$; N, 12.45; found $\mathrm{C}, 71.15$; Journal of Young Pharmacists Vol 7 •Issue 2 • Apr-Jun 2015 
H, 6.71; N, 12.44. MS (ESI): m/z $338.1(\mathrm{M}+1)^{+}$.

1-(4-(3-Methylbenzoyl)piperazin-1-yl)-2-(phenylamino) ethanone $(6 \mathrm{~g})$

${ }^{1} \mathrm{H}$ NMR $\left(\mathrm{CDCl}_{3}\right) \delta: 7.18(\mathrm{~m}, 6 \mathrm{H}), 6.68(\mathrm{t}, J=7.3 \mathrm{~Hz}, 1 \mathrm{H})$, $6.57(\mathrm{~d}, J=7.8 \mathrm{~Hz}, 2 \mathrm{H}), 3.85(\mathrm{~s}, 2 \mathrm{H}), 3.62(\mathrm{~m}, 4 \mathrm{H}), 3.40$ (m, $4 \mathrm{H}), 2.32(\mathrm{~s}, 3 \mathrm{H})$. Anal. calcd for $\mathrm{C}_{20} \mathrm{H}_{23} \mathrm{~N}_{3} \mathrm{O}$ C, 71.19; $\mathrm{H}, 6.87$; N, 12.45; found $\mathrm{C}, 71.15 ; \mathrm{H}, 6.71 ; \mathrm{N}, 12.44$. MS (ESI): $\mathrm{m} / \mathrm{z} 338.1(\mathrm{M}+1)^{+}$.

\section{1-(4-(4-Methylbenzoyl)piperazin-1-yl)-2-(phenylamino) ethanone (6h)}

${ }^{1} \mathrm{H}$ NMR $\left(\mathrm{CDCl}_{3}\right)$ ): $7.25(\mathrm{~m}, 2 \mathrm{H}), 7.16(\mathrm{~m}, 2 \mathrm{H}), 7.12(\mathrm{~m}$, $2 \mathrm{H}), 6.68(\mathrm{t}, J=5.2 \mathrm{~Hz}, 1 \mathrm{H}), 6.57(\mathrm{~d}, J=8.0 \mathrm{~Hz}, 2 \mathrm{H})$, $3.85(\mathrm{~s}, 2 \mathrm{H}), 3.63(\mathrm{~m}, 4 \mathrm{H}) 3.42(\mathrm{~m}, 4 \mathrm{H}), 2.33$ (s, 3H). Anal. calcd for $\mathrm{C}_{20} \mathrm{H}_{23} \mathrm{~N}_{3} \mathrm{O}_{2} \mathrm{C}, 71.19 ; \mathrm{H}, 6.87 ; \mathrm{N}, 12.45$; found C, 71.15; H, 6.81; N, 12.44. MS (ESI): m/z $338.2(\mathrm{M}+1)^{+}$.

\section{1-(4-(2-Methoxybenzoyl)piperazin-1-yl)-2-} (phenylamino)ethanone (6i)

${ }^{1} \mathrm{H}$ NMR $\left(\mathrm{CDCl}_{3}\right)$ 8: $7.41(\mathrm{t}, J=7.4 \mathrm{~Hz}, 1 \mathrm{H}), 7.22(\mathrm{~m}, 3 \mathrm{H})$, $7.04(\mathrm{t}, J=7.4 \mathrm{~Hz}, 1 \mathrm{H}), 6.94$ (d, $J=8.0 \mathrm{~Hz}, 1 \mathrm{H}), 6.74$ $(\mathrm{m}, 1 \mathrm{H}), 6.64(\mathrm{~m}, 2 \mathrm{H}), 3.97(\mathrm{~s}, 2 \mathrm{H}), 3.86(\mathrm{~s}, 3 \mathrm{H}), 3.64(\mathrm{~m}$, $4 \mathrm{H}), 3.34(\mathrm{~m}, 4 \mathrm{H})$. Anal. calcd for $\mathrm{C}_{20} \mathrm{H}_{23} \mathrm{~N}_{3} \mathrm{O}_{3} \mathrm{C}$, 67.97; $\mathrm{H}, 6.56 ; \mathrm{N}, 11.89$; found $\mathrm{C}, 67.87 ; \mathrm{H}, 6.54 ; \mathrm{N}, 11.84$. MS (ESI): $\mathrm{m} / \mathrm{z} 354.1(\mathrm{M}+1)^{+}$.

\section{1-(4-(3-Methoxybenzoyl)piperazin-1-yl)-2 - (phenylamino)ethanone (6j)}

${ }^{1} \mathrm{H}$ NMR (CDCl3) $\delta: 7.27(\mathrm{t}, J=8.0 \mathrm{~Hz}, 1 \mathrm{H}), 7.13$ (m, $2 \mathrm{H}), 6.88(\mathrm{~m}, 3 \mathrm{H}), 6.67(\mathrm{t}, J=7.2 \mathrm{~Hz}, 1 \mathrm{H}), 6.56(\mathrm{~d}, J=$ $8.0 \mathrm{~Hz}, 2 \mathrm{H}), 3.85$ (s, 2H), 3.76 (s, $3 \mathrm{H}), 3.70(\mathrm{~m}, 4 \mathrm{H}), 3.45$ (m, $4 \mathrm{H})$. Anal. calcd for $\mathrm{C}_{20} \mathrm{H}_{23} \mathrm{~N}_{3} \mathrm{O}_{3} \mathrm{C}, 67.97 ; \mathrm{H}, 6.56 ; \mathrm{N}$, 11.89; found C, 67.98; H, 6.54; N, 11.81. MS (ESI): $\mathrm{m} / \mathrm{z}$ $354.1(\mathrm{M}+1)^{+}$.

\section{1-(4-(4-Methoxybenzoyl)piperazin-1-yl)-2- (phenylamino)ethanone (6k)}

${ }^{1} \mathrm{H}$ NMR $\left(\mathrm{CDCl}_{3}\right)$ 8: $7.43(\mathrm{~m}, 2 \mathrm{H}), 7.23(\mathrm{~m}, 2 \mathrm{H}), 6.96(\mathrm{~m}$, $2 \mathrm{H}), 6.77(\mathrm{t}, J=7.4 \mathrm{~Hz}, 1 \mathrm{H}), 6.66(\mathrm{~d}, J=3.6 \mathrm{~Hz}, 2 \mathrm{H})$, $3.95(\mathrm{~s}, 2 \mathrm{H}), 3.87(\mathrm{~s}, 3 \mathrm{H}), 3.73(\mathrm{~m}, 4 \mathrm{H}), 3.66(\mathrm{~m}, 2 \mathrm{H}), 3.54$ (m, 2H). Anal. calcd for $\mathrm{C}_{20} \mathrm{H}_{23} \mathrm{~N}_{3} \mathrm{O}_{3} \mathrm{C}, 67.97 ; \mathrm{H}, 6.56 ; \mathrm{N}$, 11.89; found C, 67.98; $\mathrm{H}, 6.53 ; \mathrm{N}, 11.84$. MS (ESI): $\mathrm{m} / \mathrm{z}$ $354.1(\mathrm{M}+1)^{+}$.

\section{1-(4-(2-Ethylbenzoyl)piperazin-1-yl)-2-(phenylamino) ethanone (6I)}

${ }^{1} \mathrm{H}$ NMR $\left(\mathrm{CDCl}_{3}\right)$ ס: $7.39(\mathrm{~m}, 1 \mathrm{H}), 7.34(\mathrm{~m}, 1 \mathrm{H}), 7.22(\mathrm{~m}$, $2 \mathrm{H}), 7.13(\mathrm{~d}, J=7.4 \mathrm{~Hz}, 1 \mathrm{H}), 6.94(\mathrm{t}, J=8.2 \mathrm{~Hz}, 1 \mathrm{H}), 6.79$ $(\mathrm{m}, 1 \mathrm{H}), 6.67(\mathrm{~m}, 2 \mathrm{H}), 3.90(\mathrm{~s}, 2 \mathrm{H}), 3.78(\mathrm{~m}, 4 \mathrm{H}), 3.60(\mathrm{~m}$, $2 \mathrm{H}), 3.52(\mathrm{~m}, 2 \mathrm{H}), 2.79(\mathrm{q}, J=7.6 \mathrm{~Hz} 2 \mathrm{H}), 1.24(\mathrm{t}, J=7.6$ $\mathrm{Hz}, 3 \mathrm{H})$. Anal. calcd for $\mathrm{C}_{21} \mathrm{H}_{25} \mathrm{~N}_{3} \mathrm{O}_{2} \mathrm{C}, 71.77 ; \mathrm{H}, 7.17$; N, Journal of Young Pharmacists Vol 7 • Issue 2 • Apr-Jun 2015
11.96; found C, 71.68; H, 7.11; N, 11.91; MS (ESI): m/z $352.2(\mathrm{M}+1)^{+}$.

1-(4-(3-Ethylbenzoyl)piperazin-1-yl)-2-(phenylamino) ethanone $(6 \mathrm{~m})$

${ }^{1} \mathrm{H}$ NMR $\left(\mathrm{CDCl}_{3}\right) \delta: 7.27(\mathrm{t}, J=8.0 \mathrm{~Hz}, 1 \mathrm{H}), 7.13(\mathrm{~d}, J=$ $7.2 \mathrm{~Hz}, 1 \mathrm{H}), 6.88(\mathrm{~m}, 4 \mathrm{H}), 6.67(\mathrm{~m}, 1 \mathrm{H}), 6.56(\mathrm{~d}, J=8.0$ $\mathrm{Hz}, 2 \mathrm{H}), 3.84$ (s, 2H), 3.68 (m, 4H), 3.55 (m, 4H), 2.78 (q, $J=7.6 \mathrm{~Hz} 2 \mathrm{H}), 1.28$ (t, $J=7.6 \mathrm{~Hz}, 3 \mathrm{H}$ ). Anal. calcd for $\mathrm{C}_{21} \mathrm{H}_{25} \mathrm{~N}_{3} \mathrm{O}_{2} \mathrm{C}, 71.77$; H, 7.17; N, 11.96; found C, 71.77; $\mathrm{H}, 7.20$; N, 11.84; MS (ESI): $\mathrm{m} / \mathrm{z} 352.2(\mathrm{M}+1)^{+}$.

\section{1-(4-(4-Ethylbenzoyl)piperazin-1-yl)-2-(phenylamino) ethanone (6n)}

${ }^{1} \mathrm{H} \mathrm{NMR}\left(\mathrm{CDCl}_{3}\right)$ ): $7.37(\mathrm{~d}, J=7.8 \mathrm{~Hz}, 2 \mathrm{H}), 7.28(\mathrm{~m}, 2 \mathrm{H})$, $7.22(\mathrm{t}, J=7.8 \mathrm{~Hz}, 2 \mathrm{H}), 6.76(\mathrm{t}, J=7.4 \mathrm{~Hz}, 1 \mathrm{H}), 6.65$ (d, $J=7.6 \mathrm{~Hz}, 2 \mathrm{H}), 3.94(\mathrm{~s}, 2 \mathrm{H}), 3.72(\mathrm{~m}, 4 \mathrm{H}), 3.53(\mathrm{~m}, 4 \mathrm{H})$, 2.71 (q, $J=7.6 \mathrm{~Hz} 2 \mathrm{H}), 1.28$ (t, $J=7.6 \mathrm{~Hz}, 3 \mathrm{H})$. Anal. calcd for $\mathrm{C}_{21} \mathrm{H}_{25} \mathrm{~N}_{3} \mathrm{O}_{2} \mathrm{C}, 71.77 ; \mathrm{H}, 7.17 ; \mathrm{N}, 11.96$; found C, 71.78; H, 7.19; N, 11.94; MS (ESI): m/z $352.2(\mathrm{M}+1)^{+}$.

\section{1-(4-(2-Ethoxybenzoyl)piperazin-1-yl)-2-(phenylamino)} ethanone (60)

${ }^{1} \mathrm{H}$ NMR $\left(\mathrm{CDCl}_{3}\right) \delta: 7.39(\mathrm{~m}, 1 \mathrm{H}), 7.30(\mathrm{~m}, 1 \mathrm{H}), 7.24(\mathrm{~m}$, $2 \mathrm{H}), 7.03(\mathrm{t}, J=7.4 \mathrm{~Hz}, 1 \mathrm{H}), 6.94(\mathrm{~d}, J=8.2 \mathrm{~Hz}, 1 \mathrm{H})$, $6.79(\mathrm{~m}, 1 \mathrm{H}), 6.67(\mathrm{~m}, 2 \mathrm{H}), 4.10(\mathrm{q}, J=7.4 \mathrm{~Hz}, 2 \mathrm{H}), 3.98$ $(\mathrm{s}, 2 \mathrm{H}), 3.90(\mathrm{~m}, 1 \mathrm{H}), 3.71(\mathrm{~m}, 2 \mathrm{H}), 3.58(\mathrm{~m}, 2 \mathrm{H}), 3.42(\mathrm{~m}$, $2 \mathrm{H}), 3.28(\mathrm{~m}, 1 \mathrm{H}), 1.41(\mathrm{t}, J=7.4 \mathrm{~Hz}, 3 \mathrm{H})$. Anal. calcd for $\mathrm{C}_{21} \mathrm{H}_{25} \mathrm{~N}_{3} \mathrm{O}_{3} \mathrm{C}$, 68.64; $\mathrm{H}, 6.86$; N, 11.44; found C, 68.62; $\mathrm{H}, 6.84 ; \mathrm{N}, 11.40 ; \mathrm{MS}$ (ESI): $\mathrm{m} / \mathrm{z} 368.2(\mathrm{M}+1)^{+}$.

\section{1-(4-(3-Ethoxybenzoyl)piperazin-1-yl)-2-(phenylamino) ethanone (6p)}

${ }^{1} \mathrm{H}$ NMR $\left(\mathrm{CDCl}_{3}\right)$ ): $7.26(\mathrm{~m}, 1 \mathrm{H}), 7.13(\mathrm{~m}, 2 \mathrm{H}), 6.91(\mathrm{~m}$, $1 \mathrm{H}), 6.89(\mathrm{~m}, 1 \mathrm{H}), 6.82(\mathrm{~m}, 1 \mathrm{H}), 6.67(\mathrm{t}, J=5.4 \mathrm{~Hz}, 1 \mathrm{H})$, $6.56(\mathrm{~d}, J=8.0 \mathrm{~Hz}, 2 \mathrm{H}), 3.98(\mathrm{q}, J=7.0 \mathrm{~Hz}, 2 \mathrm{H}), 3.85(\mathrm{~s}$, $2 \mathrm{H}), 3.62(\mathrm{~m}, 2 \mathrm{H}), 3.41(\mathrm{~m}, 6 \mathrm{H}), 1.36(\mathrm{t}, J=7.0 \mathrm{~Hz}, 3 \mathrm{H})$. Anal. calcd for $\mathrm{C}_{21} \mathrm{H}_{25} \mathrm{~N}_{3} \mathrm{O}_{3} \mathrm{C}, 68.64 ; \mathrm{H}, 6.86 ; \mathrm{N}, 11.44$; found $\mathrm{C}, 68.61 ; \mathrm{H}, 6.84 ; \mathrm{N}, 11.41$; MS (ESI): m/z 368.1 $(\mathrm{M}+1)^{+}$.

\section{1-(4-(4-Ethoxybenzoyl)piperazin-1-yl)-2-(phenylamino)} ethanone (6q)

${ }^{1} \mathrm{H} \mathrm{NMR}\left(\mathrm{CDCl}_{3}\right)$ 8: $7.41(\mathrm{~m}, 2 \mathrm{H}), 7.23(\mathrm{~m}, 2 \mathrm{H}), 6.95(\mathrm{~m}$, $2 \mathrm{H}), 6.78(\mathrm{t}, J=7.4 \mathrm{~Hz}, 1 \mathrm{H}), 6.68(\mathrm{~d}, J=7.6 \mathrm{~Hz}, 2 \mathrm{H}), 4.10$ $(\mathrm{q}, J=6.8 \mathrm{~Hz}, 2 \mathrm{H}), 3.95(\mathrm{~s}, 2 \mathrm{H}), 3.73(\mathrm{~m}, 2 \mathrm{H}), 3.66(\mathrm{~m}$, $2 \mathrm{H}), 3.50(\mathrm{~m}, 4 \mathrm{H}), 1.46(\mathrm{t}, J=6.8 \mathrm{~Hz}, 3 \mathrm{H})$. Anal. calcd for $\mathrm{C}_{21} \mathrm{H}_{25} \mathrm{~N}_{3} \mathrm{O}_{3} \mathrm{C}$, 68.64; $\mathrm{H}, 6.86$; N, 11.44; found C, 68.63; $\mathrm{H}, 6.84$; N, 11.43; MS (ESI): m/z $368.2(\mathrm{M}+1)^{+}$.

\section{Enzyme assay}

The inhibitory potency of seventeen novel molecules 
were analyzed by their ability to block the activity of falcipain-2. The protocol for the purification and refolding of recombinant protein falcipain- $2^{19,20}$ was followed. The assays were performed in 96-well plates, following a protocol described. ${ }^{21}$ In short, a mixture containing 10 mM DTT, $100 \mathrm{mM} \mathrm{NaOAc}, 6 \mu \mathrm{M}$ of the enzyme and different concentrations of inhibitors, $\mathrm{pH} 5.5,10 \mathrm{mM}$ of fluorogenic substrate benzyloxycarbonyl-Phe-Arg7-amino-4-methylcoumarin hydrochloride (ZFR-AMC) was added. The FP-2 activity is assessed by release of 7-amino-4- methylcoumarin (AMC) (excitation $355 \mathrm{~nm}$; emission $460 \mathrm{~nm}$ ) over $30 \mathrm{~min}$ at RT in Perkin Elmer Victor3 multi-label counter. Change in fluorescence intensity in a sample indicates inhibition of enzyme activity. The $\mathrm{IC}_{50}$ values were calculated based on curve fittings by software workout V 2.5.

The enzyme, remaining activity was determined as a percentage of the uninhibited reaction. The remaining activity data was plotted versus inhibitor concentration and fitted to the dose-response equation to compute $\mathrm{IC}_{50}$. The data points were fitted to the following equation:

$$
y=\frac{\text { Range }}{1+\left(\frac{X}{I C_{50}}\right)^{5}} \quad \ldots . . \text { Equation } 1
$$

In this equation, Range is the fitted uninhibited value, $s$ is a slope factor.

\section{Docking Studies}

Molecular modeling studies are required to generate molecular models that assimilate all experimental evidences reported. Considering the well retrieved in vitro results, it was thought worthy to perform in silico studies for all newly synthesized molecules 6(a-q). Glide v5.9 (Schrodinger, Inc.) running on maestro version 9.4, was used to investigate the binding mode of the molecules. ${ }^{22}$ The crystal structure of falcipain-2 (FP-2) was obtained from the Protein Data Bank (PDB entry: 3bpf) with a resolution of $2.9 \mathrm{~A}^{\circ}$. The conserved catalytic site residues of Cys 42, Asn 173 and His 174 is located in a junction between the structurally distinct domains. ${ }^{23}$ Gln 36, Ser 41 and Asn 81 are conserved amino acids participate in the formation of additional hydrogen bond with substrate. ${ }^{15}$ Initially, the typical steps (i) addition of hydrogen atoms and atomic charges, (ii) elimination of crystallographic water molecules from the coordinate set, using the Protein Preparation Wizard in Maestro v9.4 were included in the docking procedure. Energy was minimized with Root Mean Square Deviation (RMSD) value of 0.3 $\AA$ using Optimized Potentials for Liquid Simulations-2005 (OPLS-2005) force field. ${ }^{24}$ Potential of non-polar parts of receptors was softened by scaling van der Walls radii of ligand atoms by $1.00 \AA$ to generate the grid. The initial $3 \mathrm{D}$ structures of the analogues $6(\mathrm{a}-\mathrm{q})$ were generated using chemsketch and optimized with the help of LigPrep module. In the docking process, extra-precision (XP) docking mode was adopted to generate the minimized poses, and the Glide scoring function (G-Score) was referred to select at most ten poses for compounds $6(\mathrm{a}-\mathrm{q})$ upon visualized observation.

\section{RESULTS}

Based on our designed three component pharmacophore model, a novel series 1-(4-(substituted)piperazin-1-yl)-2(phenylamino)ethanone derivatives as falcipain-2 inhibitors were designed and synthesized. All newly synthesized compounds $6(\mathrm{a}-\mathrm{q})$ were evaluated for their in vitro inhibitor activities against falcipain-2 enzyme (FP-2) using the fluorometric assay and the results are summarized in Table 1. Results obtained from the screening of these derivatives against falcipain-2 enzyme indicates that, compounds

\begin{tabular}{|c|c|c|c|c|c|}
\hline Compound & $\mathbf{R}$ & $I C_{w}(\mu \mathrm{M})$ & Compound & $\mathbf{R}$ & $\mathrm{IC}_{50}(\mu \mathrm{M})$ \\
\hline $6 a$ & $-\mathrm{H}$ & 80.2 & $6 j$ & $m-\mathrm{OCH}_{3}$ & 75.1 \\
\hline $6 b$ & $o-F$ & 90.7 & $6 k$ & $p-\mathrm{OCH}_{3}$ & 75.5 \\
\hline $6 c$ & $p-\mathrm{F}$ & NA & 61 & $\mathrm{O}-\mathrm{CH}_{2} \mathrm{CH}_{3}$ & $>100$ \\
\hline $6 d$ & $p-\mathrm{Cl}$ & NA & $6 m$ & $m-\mathrm{CH}_{2} \mathrm{CH}_{3}$ & $>100$ \\
\hline $6 e$ & $p-\mathrm{CF}_{3}$ & NA & $6 n$ & $p-\mathrm{CH}_{2} \mathrm{CH}_{3}$ & 48.7 \\
\hline $6 f$ & $\mathrm{O}-\mathrm{CH}_{3}$ & 55.6 & 60 & $\mathrm{O}-\mathrm{OCH}_{2} \mathrm{CH}_{3}$ & 49.1 \\
\hline $6 g$ & $m-\mathrm{CH}_{3}$ & 65.1 & $6 p$ & $m-\mathrm{OCH}_{2} \mathrm{CH}_{3}$ & $>100$ \\
\hline $6 \mathrm{~h}$ & $p-\mathrm{CH}_{3}$ & 45.1 & $6 q$ & $p-\mathrm{OCH}_{2} \mathrm{CH}_{3}$ & $>100$ \\
\hline $6 i$ & $\mathrm{O}-\mathrm{OCH}_{3}$ & 72.2 & - & - & - \\
\hline
\end{tabular}



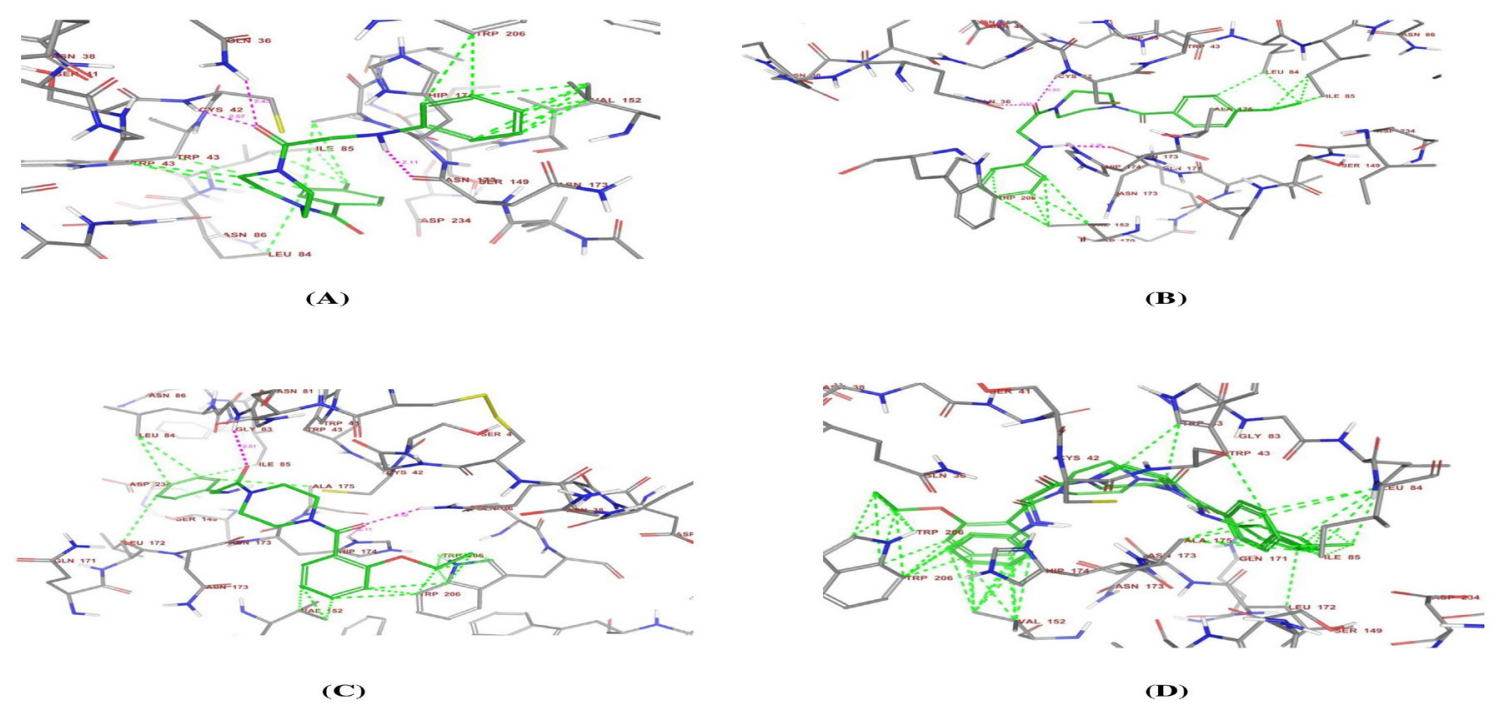

Figure 1 (A-C): Predicted binding poses of 6h, 6nand6o respectively docked tochain A offalcipain-II protein (3BPF.pdb). The red dashed line represents the possible hydrogen bond and green dashed line represents the possible hydrophobic interaction (D) Superimposition of the docked conformations of compounds $6 \mathrm{~h}, 6 \mathrm{n}$ and 60 in the active site of FP-2. All pictures are drawn using PyMol.

containing electron releasing substituents on phenyl nucleus showed weak to moderate antimalarial activity as compared to the generated pharmacophones holding with the electron withdrawing groups except compound 6b. Moreover, it was found that compounds containing methyl and ethylene groups as electron releasing substituent on phenyl nucleus at $4^{\text {th }}$ position, produced most potent compounds as evidenced by in-vitro activity results (Table 1). Overall, three compounds (6h, 6n, and 6o) showed good inhibitory activity, and among them $6 \mathrm{~h}$ was found as a most potent compound. However, to get the conclusive results, plasmodia study has to be done, which will be the futuristic extension of the work.

In silico studies revealed that all the synthesized molecules showed glide score (a model energy function) toward the target protein ranging from -5.89 to $-3.82 \mathrm{kcal} /$ mol and compounds $6 \mathrm{~h}$, 6n, and 6o showed minimum binding energies. On comparison of interacting residues of these active analogues $(6 \mathrm{~h}, 6 \mathrm{n}, 6 \mathrm{o})$ showed that, amino acids such as $\operatorname{Trp}$ 206, Ile 85, Leu 84, Val 152 were most commonly involved in hydrophobic interactions and Asn 173, Cys 42, Gln 36 amino acids involved in hydrogen bonding as shown in Figure 1 (A-D). Glide results for the top three analogues are summarized as XP Gscore,

Table 2: Docking simulations results for the most active compounds (6h, 6n and 6o) using Glide software.

\begin{tabular}{|lllll} 
Ligand & G-score & $\begin{array}{l}\text { Lipophilic } \\
\text { EvdW }\end{array}$ & H bond & $\begin{array}{l}\text { XP } \\
\text { Electro }\end{array}$ \\
\hline $6 h$ & -5.897 & -3.891 & -1.798 & -0.566 \\
\hline $\mathbf{n}$ & -5.508 & -3.743 & -1.797 & -0.529 \\
\hline 60 & -5.370 & -3.930 & -1.577 & -0.528 \\
\hline
\end{tabular}

Journal of Young Pharmacists Vol 7 • Issue 2 • Apr-Jun 2015 lipophilic energy, H-bond energy and electrophoretic energy (XP electro) in Table 2. The most potent compound 6h showed five hydrophobic interactions Trp 206, Ile 85, Leu 84, Val 152, Trp 43; three hydrogen bonds, Gln 36 $(2.43 \AA)$, Asn 173 (2.11 $\AA)$, Cys 42 (2.57 $)$. Compound 6n showed five hydrophobic interactions, $\operatorname{Trp} 206$, Ile 85, Leu 84, Val 152, Ala 175; three hydrogen bonds, Gln 36 (2.31 $\AA)$, Asn 173 (2.26 $\AA$ ), Cys 42 (2.62 A). Compound 6o showed six hydrophobic interactions, Trp 206, Ile 85, Leu 84, Val 152, Ala 175, Leu 172; three hydrogen bonds, Hip

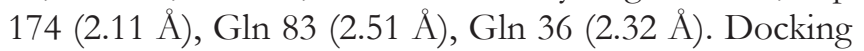
analysis revealed that hydrogen bond (HB) formation and hydrophobic interactions are the most important factors affecting inhibitory action of compounds. To validate the docking model, co-crystallized ligand E-64 was removed from the active site and subjected to redocked it back into the binding pocket of the enzyme.

\section{DISCUSSION}

The broad specificity of cysteine proteases creates a challenge for designing of inhibitors that are potentially selective in nature. ${ }^{20}$ In our drug designing strategy, we have selected few existing potential falcipain-2 inhibitors ${ }^{25,26}$ $\left(\mathrm{IC}_{50}\right.$ value range from 1 to $\left.10.9 \mu \mathrm{M}\right)$ and composed a basic core structure by mimicking with chain length which was depicted in Figure 2. The core features of the selected structures were: a) hydrophobic moiety; commonly an aromatic residue, b) aromatic moiety (monocyclic/bicyclic), and c) linker; hydrogen bond donor and acceptor atom(s), which attached the hydrophobic moiety to the aromatic residue. 


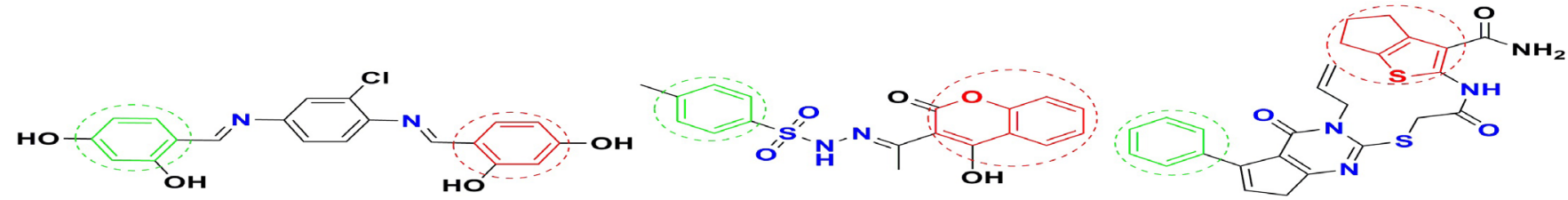

$A, 1 C_{50}=2.1 \mathrm{\mu m}$

$B, 1 C_{50}=3.9 \mu \mathrm{m}$

$C, 1 C_{50}=2.4 \mu \mathrm{m} \mathrm{Cl}$<smiles>O=C(O)C1=C(NN=Cc2ccc(C=NNc3ccccc3C(=O)O)cc2)C=C=C=C1</smiles>

$D, 1 C_{50}=1.0 \mu \mathrm{m}$

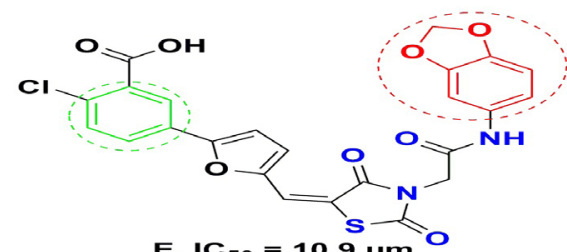

$E, 1 C_{50}=10.9 \mu \mathrm{m}$<smiles>Cc1ccc(NC(=O)C(C)Sc2nc(-c3ccc#cc3O)n[nH]2)cc1</smiles>

$F, 1 C_{50}=5.8 \mathrm{\mu m}$<smiles></smiles>

$H, I C_{50}=4.6 \mu \mathrm{m}$

Figure 2: Chemical structures of some existing falcipain-2 Inhibitors. (Green Color: Representing hydrophobic moiety; commonly an aromatic group, RedColor:Representinganaromatic residue(monocyclic/bicyclic), BlueColor:Representing thenumbersofhydrogenbonddonorandacceptoratoms).

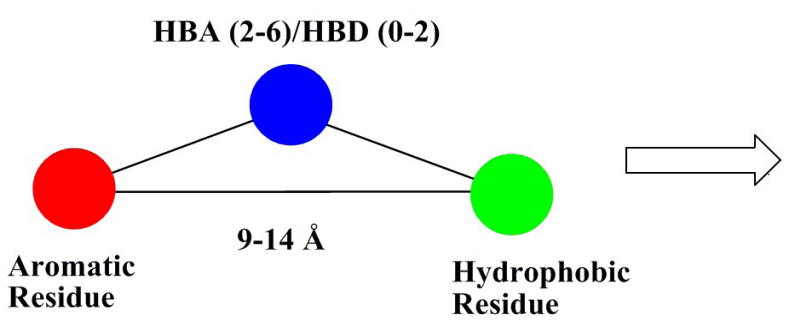

A

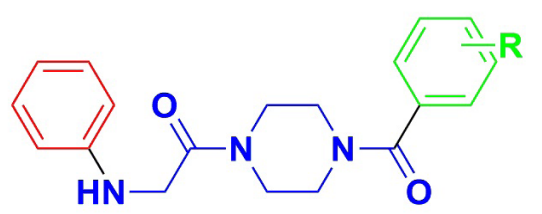

Red color: Aromatic residue as phenyl ring

Green color: Hydrophobic residue (Aromatic group)

Blue Color: Hydrogen bonding acceptor or donar atoms

Figure 3 (A-B): (A) Pharmacophore model (B) Basic structure for FP-2 inhibitors.

\begin{tabular}{|c|c|c|c|c|c|}
\hline Compound & $\mathbf{R}$ & Molecular weight & $\%$ Yield $^{c}$ & m.p. in ${ }^{\circ} \mathrm{C}$ & $\log P^{d)}$ \\
\hline $6 a$ & $-H$ & 323.3 & 58 & $141-143$ & 1.54 \\
\hline $6 b$ & $o-F$ & 341.3 & 69 & $146-149$ & 1.70 \\
\hline $6 c$ & $p-\mathrm{F}$ & 341.3 & 54 & $127-129$ & 1.70 \\
\hline $6 d$ & $p-\mathrm{Cl}$ & 357.8 & 65 & $134-136$ & 2.10 \\
\hline $6 e$ & $p-\mathrm{CF}_{3}$ & 391.3 & 61 & $130-132$ & 2.46 \\
\hline $6 f$ & $\mathrm{o}-\mathrm{CH}_{3}$ & 337.4 & 56 & $132-134$ & 2.03 \\
\hline $6 g$ & $m-\mathrm{CH}_{3}$ & 337.4 & 76 & $118-120$ & 2.03 \\
\hline $6 \mathrm{~h}$ & $p-\mathrm{CH}_{3}$ & 337.4 & 81 & $142-144$ & 2.03 \\
\hline $6 \mathrm{i}$ & $\mathrm{o}-\mathrm{OCH}_{3}$ & 353.4 & 71 & $139-141$ & 1.42 \\
\hline $6 \mathrm{j}$ & $m-\mathrm{OCH}_{3}$ & 353.4 & 62 & $122-124$ & 1.42 \\
\hline $6 k$ & $p-\mathrm{OCH}_{3}$ & 353.4 & 66 & $130-132$ & 1.42 \\
\hline 61 & $\mathrm{O}-\mathrm{CH}_{2} \mathrm{CH}_{3}$ & 351.4 & 72 & $130-132$ & 2.45 \\
\hline $6 \mathrm{~m}$ & $m-\mathrm{CH}_{2} \mathrm{CH}_{3}$ & 351.4 & 73 & $116-118$ & 2.45 \\
\hline $6 n$ & $p-\mathrm{CH}_{2} \mathrm{CH}_{3}$ & 351.4 & 75 & $144-146$ & 2.45 \\
\hline 60 & $\mathrm{o}-\mathrm{OCH}_{2} \mathrm{CH}_{3}$ & 367.4 & 62 & $124-126$ & 1.75 \\
\hline $6 p$ & $m-\mathrm{OCH}_{2} \mathrm{CH}_{3}$ & 367.4 & 67 & $120-122$ & 1.75 \\
\hline $6 q$ & $p-\mathrm{OCH}_{2} \mathrm{CH}_{3}$ & 367.4 & 58 & $122-124$ & 1.75 \\
\hline
\end{tabular}




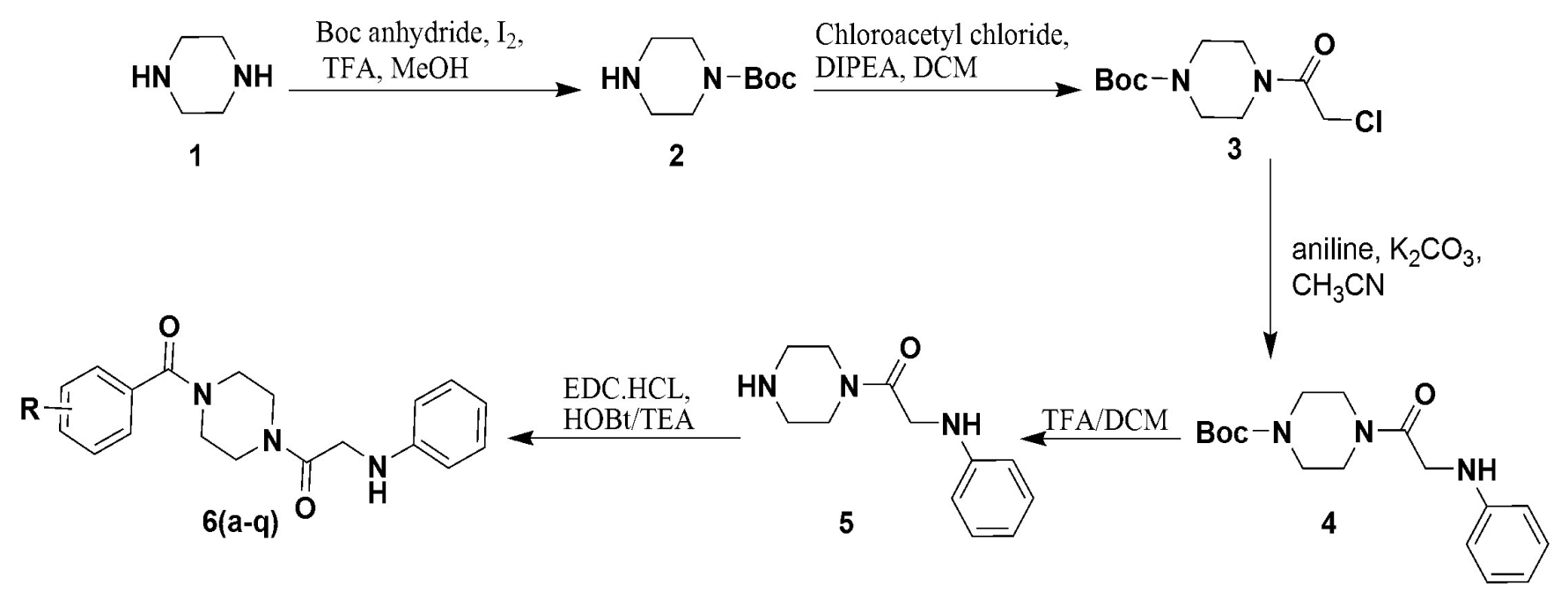

Scheme 1: Synthetic protocol for target compounds 6(a-q).

The pharmacophoric distance among the hydrophobic group and aromatic residue ranges between 9 to $14 \AA$ and Log P values of these compounds varies in between 2 to 5. The hydrogen bond acceptor (range; $2-6$ ) and hydrogen bond donor (range; 0-2) atom(s), are present as either alicyclic/heterocyclic or open chain form. After examining these common features towards the design of a basic backbone as a falcipain-2 inhibitor, a three component pharmacophore mode ${ }^{27}$ has been built which was shown in Figure 3 A. Further, a series of novel falcipain-2 inhibitors were designed and synthesized as represented by the basic structure in Figure 3 B.

Structural features such as hydrogen bond donors, hydrogen bond acceptors of the designed compounds, complied with the pharmacophoric model. The minimum energy conformation (three least energy conformations for each compound) of each designed molecule was generated by ACDLABS-10.0/3D Viewer, and the pharmacophoric distances were measured from centroid of aromatic residue to the centroid of hydrophobic residue. The computed distances between the pharmacophoric components of all the designed compounds are in accordance with the mentioned pharmacophoric model. Lipophilicity is an key parameter to be considered, while designing compound

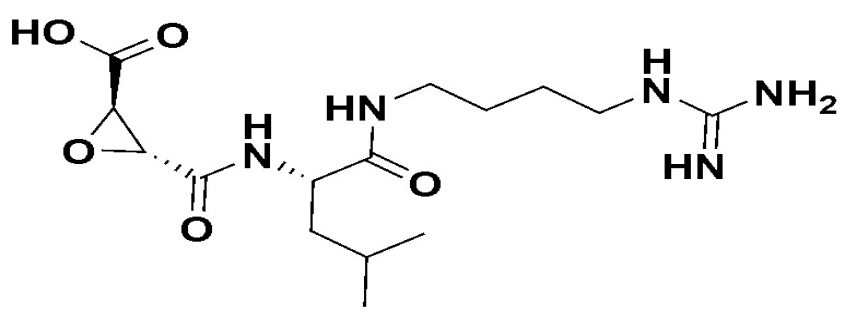

Figure 4: Structure of reference drug E-64. to display drug-like profile. Therefore, Log $\mathrm{P}$ values of all the designed compounds were computed using JME 4 Molecular Editor (Courtesy of Peter Ertl; Novartis; Table 3). Further, for better pharmacokinetics (absorption, distribution, metabolism and excretion) profile all the molecules were designed according to Lipinski's rules of five. ${ }^{28,29}$ Thus, synthesis of a series of 1-(4-(substituted) piperazin-1-yl)-2-(phenylamino)ethanone derivatives as FP-2 inhibitors were pursued with an intent to examine the anti-malarial activity of these compounds.

The target compounds were synthesized as depicted in Scheme 1. Intermediate compound 5 was synthesized in multi-gram scale from the starting material piperazine in a sequence of reactions. Initially, $N$-Boc piperazine 2 was prepared by reaction between piperazine and di-tertbutyldicarbonate. Chloroacetyl chloride was subjected to nucleophilic substitution reaction with intermediate 2, furnished the compound 3. This intermediate was reacted with aniline, which afforded compound 4 . On subsequent, deprotection of intermediate 4 with trifluoroacetic acid, the key intermediate, 2-(phenylamino)1-(piperazin-1-yl) ethanone (5) was obtained. The final compounds were synthesized from intermediate 5 , by coupling with appropriate carboxylic acids in the presence of 1-(3-dimethylaminopropyl)-3-ethylcarbodiimide hydrochloride $(\mathrm{EDC} \cdot \mathrm{HCl})$ and 1-hydroxybenzotriazole (HOBt) under nitrogen atmosphere. Synthesized compounds were isolated, purified and characterized by ${ }^{1} \mathrm{H}$ NMR, mass spectroscopy, HPLC and elemental analyses. The analytical and spectral data of the compounds were found to comply with the structure of the synthesized compounds. 
Initial screening of two compounds $6 \mathrm{a}$ and $6 \mathrm{~b}$ showed $\mathrm{IC}_{50}$ values of $80.2 \mu \mathrm{M}$ and $90.7 \mu \mathrm{M}$ respectively. E-64 ( $N$-(transEpoxysuccinyl)-L-leucine 4-guanidinobutylamide, Figure 4) was used as a reference drug in the assay. ${ }^{30} \mathrm{In}$ a bid, to see the effect of different substituent on the phenyl ring and generate structure activity relationships, a series of compounds with electron withdrawing as well as electron donating groups were introduced at different positions in the phenyl ring, synthesized and screened. The effect of the fluorine group, an electron withdrawing substituent was explored at 4 position of the phenyl ring (compound 6c), the generated compound showed no inhibition. A similar response was observed after replacement of an electron withdrawing group with chloro and trifluoromethyl substituents at 4 position of the aromatic ring (compounds $6 \mathrm{~d}$ and $6 \mathrm{e}$ respectively).

Subsequently, methyl group, a weaker electron releasing group was introduced at 2, 3 and 4 positions of the phenyl ring, the resultant compounds (6f, $6 \mathrm{~g}$ and $6 \mathrm{~h}$ ) exhibited more inhibition than the hit compound 6a. It is remarkable that the inhibitory activity of compound $6 \mathrm{~h}$ increased $\sim 2$ times than that of compound $6 \mathrm{~b}$ $\left(\mathrm{IC}_{50}\right.$ decreases from $90.7 \mu \mathrm{M}$ down to $45.1 \mu \mathrm{M}$, Table 1). Incorporation of another electron releasing methoxy group at 2, 3 and 4 positions of the phenyl ring resulted compounds (6i, $6 \mathrm{j}$ and $6 \mathrm{k}$ ) exhibited moderately improved inhibition than the compound 6a. Further, replacement of the methoxy group with its higher homologue, i.e., ethoxy group at position 2, which is compound 6o $\left(\mathrm{IC}_{50}\right.$ value $\left.49.1 \mu \mathrm{M}\right)$ showed higher potency than the hit compound 6a. However, investigation of the ethoxy group at 3 and 4 positions to get compounds 6p, 6q of the phenyl ring showed lesser potency $\left(\mathrm{IC}_{50}\right.$ value; $\left.>100 \mu \mathrm{M}\right)$, as compared to the hit compound. Although compounds 61 (ethyl at position 2) and $6 \mathrm{~m}$ (ethyl at position 3 ) had the same electron releasing group at different positions in the phenyl ring, they have showed lower potency $\left(\mathrm{IC}_{50}\right.$ value $\left.>100 \mu \mathrm{M}\right)$ compared to compound 6a. Followed by, attachment of ethyl group at 4 position (compound 6n) of the phenyl ring, improves the inhibition activity as compared to its regio-isomer compounds 61 and $6 \mathrm{~m}$.

In order to understand the difference in the activity of the hit molecule (6h) with the inactive compounds (6c, 6d and 6o), their binding mode and interaction studies of different poses were analyzed. It was found that compounds which are inactive, exhibited single hydrogen bond interaction either with Asp 173 (6c, 6d), or Gln 36 (6e), compared to the hit compound $6 \mathrm{~h}$, which showed strong hydrogen bond interaction with Asn 173, Cys 42, Gln 36 (Figure 1 A-C). Moreover, there was a significant difference in the two scoring functions, such as Lipophilic EvdW (-3.42 to $-2.95)$ and XP electro (-0.501 to -0.244) of the inactive compounds versus potent compound (Table 2), which can create a major difference in the docking score (drug-protein interactions) and may be responsible for the significant variation in biological activity.

\section{CONCLUSION}

In the present study, we reported the design, synthesis and screening of 1,4 substituted piperazine derivatives as novel falcipain-2 inhibitors. The structures of the compounds were assigned on the basis of ${ }^{1} \mathrm{H}$ NMR, mass spectroscopy and elemental analysis. From the in-vitro data, on close perlustration and analysis, it was found that three compounds (6h, 6n, and 6o) showed good inhibitory activity. Compound $6 \mathrm{~h}$ is the most potent compounds from this series and it can be used as potential lead compound in the designing of new candidates to incur clinically significant anti-malarial agents. Further, pharmacological experiments will be required to evaluate the significance of these findings to malaria infection in red blood cells both in vitro and in vivo. Thus, the present approach could be an excellent starting point for the discovery of new parasitic cysteine protease inhibitors, and in general, for the development of a new class of drugs for treating malaria.

\section{SUPPLEMENTARY DATA}

Supplementary data (purity data for final compounds) associated with this article can be found, in the online version.

\section{CONFLICT OF INTEREST}

Authors declared no conflict of interest.

\section{ACKNOWLEDGMENTS}

The authors gratefully acknowledge the financial support from Department of Biotechnology (BT/IN/Canada/22/ $A M / 2009)$, New Delhi, India as a part of ISTP CanadaDBT Collaborative R\&D Program. Authors are also thankful to Birla Institute of Technology \& Science (BITS), Pilani, India, and SAIF, Panjab University, Chandigarh, India, for providing the infrastructure facilities and analytical facilities, respectively. The authors are grateful to Dr. Asif and Dr. L. Kotra for their valuable suggestions. 


\section{REFERENCES}

1. World Health Organization; World Malaria Report, WHO Press (Geneva); 2012.

2. Wongsrichanalai C, Meshnick SR. Declining artesunatemefloquine efficacy against falciparum malaria on the CambodiaThailand border. Emerging infectious diseases 2008; 14(5): 716-9.

3. Dondorp AM, Nosten F, Yi P, Das D, Phyo AP, Tarning J, et al. Artemisinin resistance in Plasmodium falciparum malaria. The New England journal of medicine 2009; 361(5): 455-67.

4. Shenai BR, Sijwali PS, Singh A, Rosenthal PJ. Characterization of native and recombinant falcipain-2, a principal trophozoite cysteine protease and essential hemoglobinase of Plasmodium falciparum. The Journal of biological chemistry 2000; 275(37): 29000-10.

5. Dua M, Raphael P, Sijwali PS, Rosenthal PJ, Hanspal M. Recombinant falcipain-2 cleaves erythrocyte membrane ankyrin and protein 4.1. Molecular and biochemical parasitology 2001; 116(1): 95-9.

6. Stoch SA, Wagner JA, Cathepsin K. inhibitors: a novel target for osteoporosis therapy. Clinical pharmacology and therapeutics 2008; 83(1): 172-6.

7. Deacon CF. Dipeptidyl peptidase-4 inhibitors in the treatment of type 2 diabetes: a comparative review. Diabetes, obesity \& metabolism 2011; 13(1): 7-18.

8. Gills JJ, Lopiccolo J, Tsurutani J, Shoemaker RH, Best CJ, AbuAsab MS, et al. Nelfinavir, A lead HIV protease inhibitor, is a broadspectrum, anticancer agent that induces endoplasmic reticulum stress, autophagy, and apoptosis in vitro and in vivo. Clinical cancer research : an official journal of the American Association for Cancer Research 2007; 13(17): 5183-94.

9. Petrov V, Fagard R, Lijnen P. Effect of protease inhibitors on angiotensin-converting enzyme activity in human T-lymphocytes. American journal of hypertension 2000; 13(5 Pt 1): 535-9.

10. Sijwali PS, Shenai BR, Gut J, Singh A, Rosenthal PJ. Expression and characterization of the Plasmodium falciparum haemoglobinase falcipain-3. The Biochemical Journal 2001; 360(Pt 2): 481-9.

11. Sijwali PS, Rosenthal PJ. Gene disruption confirms a critical role for the cysteine protease falcipain-2 in hemoglobin hydrolysis by Plasmodium falciparum. Proceedings of the National Academy of Sciences of the United States of America 2004; 101(13): 4384-9.

12. Sijwali PS, Koo J, Singh N, Rosenthal PJ. Gene disruptions demonstrate independent roles for the four falcipain cysteine proteases of Plasmodium falciparum. Molecular and biochemical parasitology 2006; 150(1): 96-106.

13. Bailly E, Jambou R, Savel J, Jaureguiberry G. Plasmodium falciparum: differential sensitivity in vitro to $\mathrm{E}-64$ (cysteine protease inhibitor) and Pepstatin A (aspartyl protease inhibitor). The Journal of protozoology 1992; 39(5): 593-9.

14. Dluzewski AR, Rangachari K, Wilson RJ, Gratzer WB. Plasmodium falciparum: protease inhibitors and inhibition of erythrocyte invasion. Experimental parasitology 1986; 62(3): 416-22.

15. Shenai BR, Lee BJ, Alvarez-Hernandez A, Chong PY, Emal $\mathrm{CD}$, Neitz RJ, et al. Structure-activity relationships for inhibition of cysteine protease activity and development of Plasmodium falciparum by peptidyl vinyl sulfones. Antimicrobial agents and chemotherapy 2003; 47(1): 154-60.

16. Lee BJ, Singh A, Chiang P, Kemp SJ, Goldman EA, Weinhouse $\mathrm{Ml}$, et al. Antimalarial activities of novel synthetic cysteine protease inhibitors. Antimicrobial agents and chemotherapy 2003; 47(12):
3810-4.

17. Palmer JT, Rasnick D, Klaus JL, Bromme D. Vinyl sulfones as mechanism-based cysteine protease inhibitors. Journal of medicinal chemistry 1995; 38(17): 3193-6.

18. Mundra S, Kotra LP. Design of inhibitors of OD Case. Future medicinal chemistry $2014 ; 6(2): 165-77$.

19. Shenai BR, Sijwali PS, Singh A, Rosenthal PJ. Characterization of native and recombinant falcipain-2, a principal trophozoite cysteine protease and essential hemoglobinase of Plasmodium falciparum The Journal of biological chemistry 2000; 275(37): 29000-10.

20. Korde R, Bhardwaj A, Singh R, Srivastava A, Chauhan VS, Bhatnagar RK, et al. A prodomain peptide of Plasmodium falciparum cysteine protease (falcipain-2) inhibits malaria parasite development. Journal of medicinal chemistry 2008; 51(11): 3116-23.

21. Kumar A, Dasaradhi PV, Chauhan VS, Malhotra P. Exploring the role of putative active site amino acids and pro-region motif of recombinant falcipain-2: a principal hemoglobinase of Plasmodium falciparum. Biochemical and biophysical research communications 2004; 317(1): 38-45.

22. Friesner RA, Banks JL, Murphy RB, Halgren TA, Klicic JJ, Mainz DT, et al. Glide: a new approach for rapid, accurate docking and scoring. 1. Method and assessment of docking accuracy. Journal of medicinal chemistry 2004; 47(7): 1739-49.

23. Wang L, Zhang S, Zhu J, Zhu L, Liu X, Shan L, et al. Identification of diverse natural products as falcipain-2 inhibitors through structurebased virtual screening. Bioorganic \& medicinal chemistry letters 2014; 24(5): 1261-4.

24. Jorgensen WL, Maxwell DS, Tirado RJ. Development and testing of the OPLS all-atom force field on conformational energetics of organic liquids. Journal of American Chemical Society 1996; 118(45): 11225-36.

25. Desai PV, Patny A, Sabnis Y, Tekwani B, Gut J, Rosenthal P, et al. Identification of novel parasitic cysteine protease inhibitors using virtual screening. 1 . The ChemBridge database. Journal of medicinal chemistry 2004; 47(26): 6609-15.

26. Li H, Huang J, Chen L, Liu X, Chen T, Zhu J, et al. Identification of novel falcipain-2 inhibitors as potential antimalarial agents through structure-based virtual screening. Journal of medicinal chemistry 2009; 52(15): 4936-40.

27. Mahesh R, Bhatt S, Devadoss T, Jindal A, Gautam B, Pandey D. Antidepressant Potential of 5-HT3 Receptor Antagonist, N-npropyl-3-ethoxyquinoxaline-2-carboxamide (6n). Journal of young pharmacists : JYP 2012; 4(4): 235-44.

28. Lipinski CA, Lombardo F, Dominy BW, Feeney PJ. Experimental and computational approaches to estimate solubility and permeability in drug discovery and development settings. Advanced drug delivery reviews 2001; 46(1-3): 3-26.

29. Azam F, Madi AM, Ali HI. Molecular Docking and Prediction of Pharmacokinetic Properties of Dual Mechanism Drugs that Block MAO-B and Adenosine A(2A) Receptors for the Treatment of Parkinson's Disease. Journal of young pharmacists : JYP 2012; 4(3): 184-92.

30. Rizzi L, Sundararaman S, Cendic K, Vaiana N, Korde R, Sinha D, et al. Design and synthesis of protein-protein interaction mimics as Plasmodium falciparum cysteine protease, falcipain-2 inhibitors. European Journal of medicinal chemistry 2011; 46(6): 2083-90. 